\title{
Improvement of Up-Converters Linearity for Ka-Band Operation
}

\author{
E. Bertran, J. Berenguer, G. Montoro, P. L. Gilabert \\ Dpt. Signal Theory and Communications \\ Universitat Politècnica Catalunya (UPC) \\ Castelldefels (Barcelona), Spain \\ bertran@tsc.upc.edu
}

\author{
A. Cidronali \\ Dept. Electronics and Telecommunications \\ University of Firenze, \\ Firenze, Italy \\ acidronali@ing.unifi.it
}

\begin{abstract}
The application of a digital predistorter to the linearisation of a Ka-band mixer is presented herein. The general objective in the TX design is to get the best trade-off between linearity and costs. This require the most of the simplicity and possibly to take advantage from already existing device without perturbing its other functionalities.

After a presentation of the problem and its constraints, we present the digital predistorter, which has been tested and debugged by means of IEEE-488 bus (GPIB) controllable instrumentation (EMA, electronic measuring automation) in order to finally consider its implementation on a fast newgeneration FPGA device. Results show an increment of the linear zone in the up-converter AM-AM curve, of about $15 \mathrm{~dB}$.
\end{abstract}

Keywords - Intermodulation distortion (IMD), Linearity, Mixers, Predistorters, Up-converters.

\section{INTRODUCTION}

The saturation of the radioelectric spectrum has boosted standardized communication services in millimeter bands. Examples are broadband LMDS (Local to Multipoint Distribution Service), which applications include metropolitan multimedia distribution, or HAPs (High Altitude Platforms) communications, which constitute an alternative for conventional satellites, mainly in wide metropolitan areas (around 100Km). In particular, the International Telecommunication Union (ITU) endorsed various frequency ranges for HAPs applications in WRC-97 (World Radio Conference, 1997). The bands 47.2-47.5 GHz and 47.9-48.2 $\mathrm{GHz}$ were allocated for fixed services, and the frequency range 18-32 GHz was allocated to a number of countries in Region 3 (Asia and Oceania) for broadband wireless applications. Further, in year 2000 (WRC-2000, Istanbul), these frequency bands were ratified. On the other hand, the new standard IEEE 802.16-SC, planned for the $10-60 \mathrm{GHz}$ range, has revived the interest on commercial $30 \mathrm{GHz}$ LMDS band (Ka band).

At these frequencies, microwave devices are sensibly expensive, especially at medium and high power levels. Hence, the cost of the equipment becomes one of the main stoppers for industry investment in millimetre bands, and consequently for applications exploitation. In the case of amplifiers, the most expensive specification is the output power level. E.g, a Kaband $32 \mathrm{dBm}$ power amplifier may cost between 2000 and $8000 \$$, approx., depending on the used technology and the kind of manufacturing process (substrate, wire-bonding, die attach, connectorization...). Regarding the cost of the power devices and the typical decrease of the power gain and the maximum available linear output power with frequency, power amplifiers for millimetre bands are usually designed making the device operating at the upper limit of its capability, preventing any possibility of adaptation of its bias conditions. Being the latter a common technique in the lower frequency range which is not possible to exploit in the frequency range under consideration..

Hence, in the transmitter design, it is very convenient to force the subsystems previous to the power amplifier to operate at their maximum linear gains, aiming at reducing the need for additional amplification in the final power amplifier. A direct consequence is the enlargement of intermediate signal levels, risking the entrance in nonlinear zones within the dynamic margins of the different subsystems.

To exploit the maximum of the devices dynamic range means to work near the compression point (low back-off operation). In LMDS or HAPs applications, were high data rate is pursuit, the adopted modulation schemes are multilevel, thus showing high PAPR (Peak-to Average Power Ratio). Consequently, to work at reduced back-off levels clips the modulated signal envelope, resulting in a lower data throughput (BER augmentation). Besides, the reduced backed-off operation follows on nonlinear effects, producing both in-band distortion (EVM augmentation) and out-of-band spurious emission, degrading the ACPR (Adjacent Channel Power Ratio), which has to be compliant with standardized values. Conversely, to enlarge the back-off value increases both signal quality and linearity degree, but at the price of a loss in power efficiency. This loss is an outstanding problem in applications using mobile handsets or solar-powered devices, as is the case of HAPs. Besides, once enlarged the back-off, it is necessary to redesign a larger compression point $(\mathrm{P} 1 \mathrm{~dB})$ device in order to keep the initially specified output power. An alternative to trade with this compromise between linearity and power 
efficiency is the use of linearizers which, reducing the nonlinear effects, allow to work at efficient back-off values. Linearizers have been mainly employed in power amplifiers, and, in minor emphasis, in mixers.

The inherent non-linearity of mixers produces both in-band and out-of-band undesired effects. Some of these effects may be eliminated by filtering, as is the case of harmonic distortion, while other effects such as intermodulation distortion (IMD, caused by odd-order nonlinearities) cannot be removed by simply filtering., thus degenerating in-band EVM and creating co-channel interference. A mixer with high IP3 and P1 dB would be chosen to achieve a large dynamic signal range. Nevertheless, this choice would increase the mixer conversion loss to an unacceptable level.

Alternatively, the effects due to mixer's nonlinearities can be reduced using a linearization method, in a straightforward application of the structures employed in power amplifiers. Some already published solutions for mixer linearization are based on the feedforward technique and other ones also based in signal cancellation loops, [1][2][3], on analog predistortion [4], or on some kind of signal injection [5], which may require a second mixer or a delicate device adjustment (if not both simultaneously). Moreover, not all of the linearizing structures are suitable for operation at millimeter bands [6].

In this paper we study and experiment a DSP-based linearizer, properly suited for power amplifiers, to improve the up-converter (UC) linearity. In particular, we apply a digital predistorter to the waveguide UC employed in a HAP application working at $30 \mathrm{GHz}$ band, aiming to enlarge the UC linear signal dynamic range. In order to cope with existing digital infrastructure (cost minimization), the linearizer has to follow a simple and not DSP time-consuming strategy.

\section{DESCRIPTION OF THE UP CONVERTER}

In our transmitter, the reference signal for different up and down-conversion (the latter is just for further power amplifier linearization, so it will no be considered here) processes is obtained from a PLO (Phase Locked Oscillator) at $28 \mathrm{GHz}$ (see Fig. 1). Its output $(20 \mathrm{dBm})$ feeds a wideband, high directivity waveguide (WR-28) coupler, with frequency range of 26.5-40 $\mathrm{GHz}$ and coupling value of $3 \mathrm{~dB}$ at centre frequency. Its directivity is $37 \mathrm{~dB}$. In the extremes of the coupler there are two waveguide balanced mixers, used for up \& down conversion from/to $2 \mathrm{GHz}$ (IF) to/from $30 \mathrm{GHz}$ (RF). The mixer used as up-converter is followed by a single sideband filter. Finally, the waveguide terminations are adapted to a 2.4 mm connector.

The original AM-AM curve of the UC is shown in Fig, 2. This UC drives a power amplifier of $33 \mathrm{dBm}$, with a gain of 15 $\mathrm{dB}$. Hence, we are forced to amplify the up-converter output signal. Anyway, the closer we can work to the UC compression point (low mixer attenuation), the lower power amplifier gain we will need. So we plan to linearize the UC.

Working at Pin $=0 \mathrm{dBm}$, and exciting the unlinearized upconverter with a two tone signal, composed by two tones separated $50 \mathrm{KHz}$ around $2 \mathrm{GHz}$, the intermodulated (IMD) up-converter output is as shown in Fig. 3

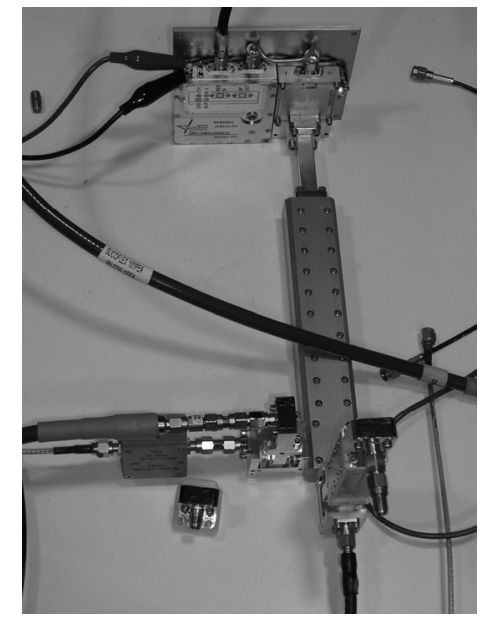

Fig. 1. Up\&down converter subsystem $(30 \mathrm{GHz})$.

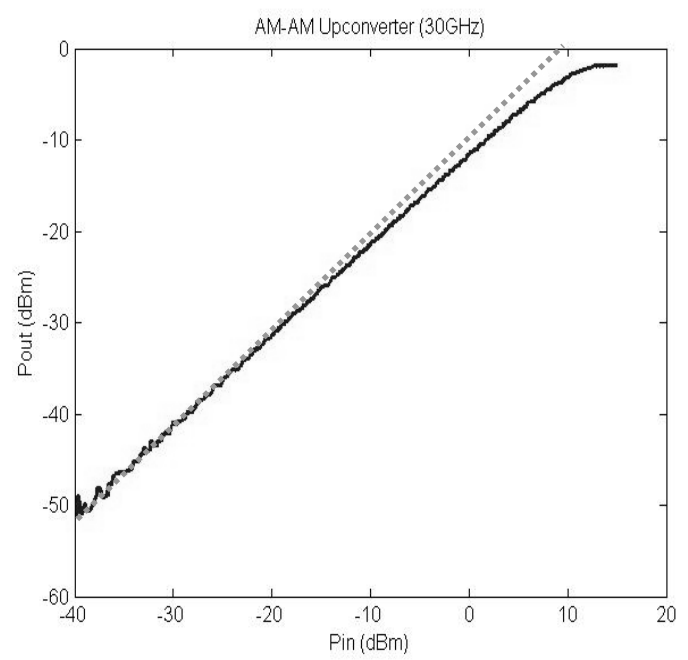

Fig. 2. AM-AM conversion curves of the up-converter (measured at $30 \mathrm{GHz}$ ). The dashed line is just for comparison with a linear reference $(\mathrm{IF}=2 \mathrm{GHz})$.

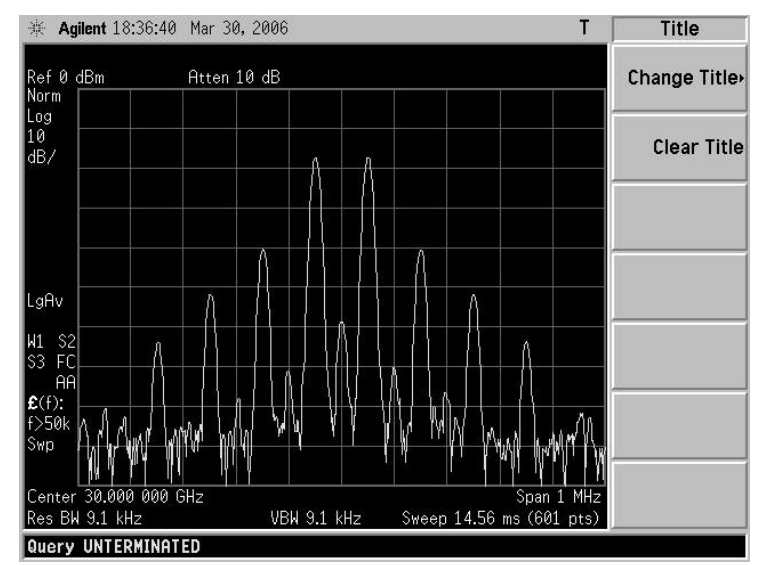

Fig. 3. IMD products in the up-converter output. Input was fixed at $0 \mathrm{dBm}$, Two tone test: $\pm 50 \mathrm{KHz} @ 2 \mathrm{GHz}$ 


\section{PREDISTORTER DESIGN \& IMPLEMENTATION}

We have selected a basic memoryless baseband digital predistorter [7]. This structure follows the block diagram shown in Fig.4, and its most common application is to linearise a power amplifier. From a straightforward adaptation, it will be here used as up-converter (UC) linearizer.

If the structure of Fig. 4 is completed with a power detector for monitoring the power of output signal $y(t)$, then it is possible to continuously characterize the static distortion characteristics. This allows the adaptation (updating) of the AM-AM curves according to variations of the UC behaviour, which may be due to reasons such as aging, temperature, impedance mismatches, etc. In the non-adaptive case, AM-AM curves are initially measured in the design process, by using a suitable spectrum analyzer, and results are used to feed the fixed values of the predistorter coefficients.

In general, the application of the memoryless baseband digital predistorters may use both AM/AM and AM/PM characterization curves. But in $\mathrm{Ka}$ band the phase measurements are difficult to be made at reduced cost or, by using digital alternatives, at an appropriate (low) time-scale (apart from A/D converters speed). Hence, only AM/AM characteristics will be here considered.

Some studies, focused on power amplifiers, considered more complex models, including memory effects: Volterra, Wiener or Hammerstein models are usual tools to do that [8]. However, our application, which intends to linearize the upconverter at Ka-band, has to be allocated without significantly additional expenditure, in coherence with the abovementioned objective to reduce further amplification costs. This obligates to allocate the digital predistorter in an already existing DSP or FPGA device, already necessary for other functionalities in the transmitter, such as data coding, modulation, interleaving and so on. In consequence the predistorter implementation has to consume few computing time in order do not enlarge the sampling interval out of the permissible (Nyquist or subNyquist rate conditions) limits. Regarding the additional needs for fast predistorter parameter's reading and updating, a suitable solution is the use of a LUT (Look Up Table) implemented in a FPGA device. Other memoryless solutions [9] based on analytical predistorters which aims at the inversions of some static model, are here not considered because our implementation restrictions (in economy and computing time).

Besides, the number of points to be allocated in the LUT has to be small enough to reduce the necessary amount of memory, and the interpolation of the values between consecutive LUT points has to follow a not time-consuming algorithm. Consequently, we have experimented a simple but efficient algorithm based on the measurement, inversion and linear interpolation of points of the up-converter $\mathrm{AM} / \mathrm{AM}$ distortion curve.

The predistorter algorithm has been evaluated using GPIB accessible generators (vectorial and $\mathrm{CW}$ ) as well as a $50 \mathrm{GHz}$ spectrum analyser. Fig. 5 shows a diagram of the lab testbench: all the instruments are interconnected using a GPIB board (USB connected to a PC). The control software has been developed in Matlab, running under Windows XP. Once evaluated and debugged, the final implementation has been considered in an Xtreme DSP Virtex-IV. It consist of a VirtexIV FPGA that controls two 14 bits A/D and D/A converters of 105 Msamples/sec. Fig. 6 shows the board. The behaviour of an updateable LUT based predistorter in this kind of device is possible maintaining the full rate signal acquisition, 105 Msamples/sec.

In fig. 7 it is shown a comparative of the AM-AM curves of the up-converter with and without predistorters. Notice the enlargement of the linear gain for input power levels comprised between -5 to $10 \mathrm{dBm}$.

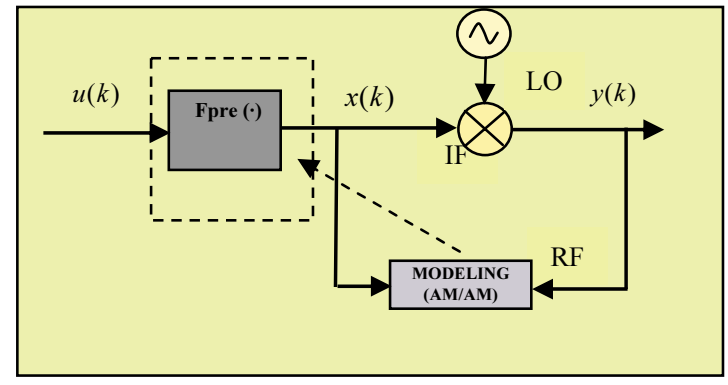

Fig.4. Digital predistortion (up-converter).

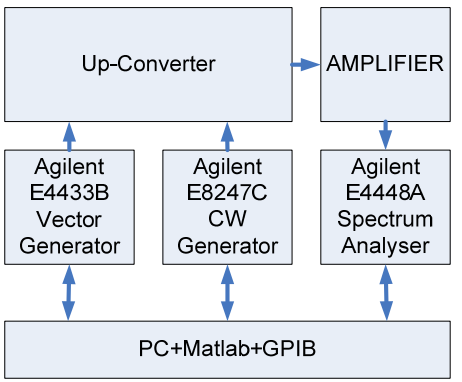

Fig.5. Linearizer testbench.

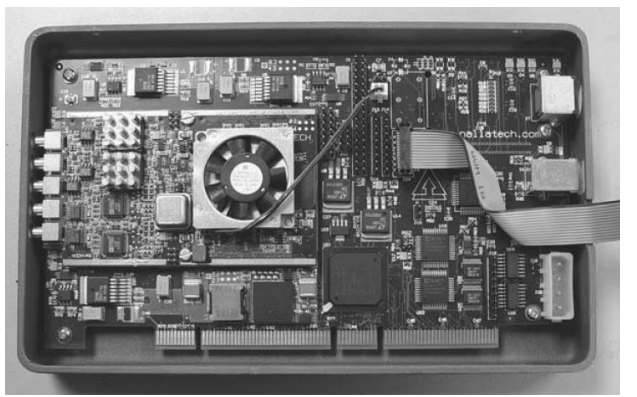

Fig.6. Xtreme DSP Virtex-IV physical board. 


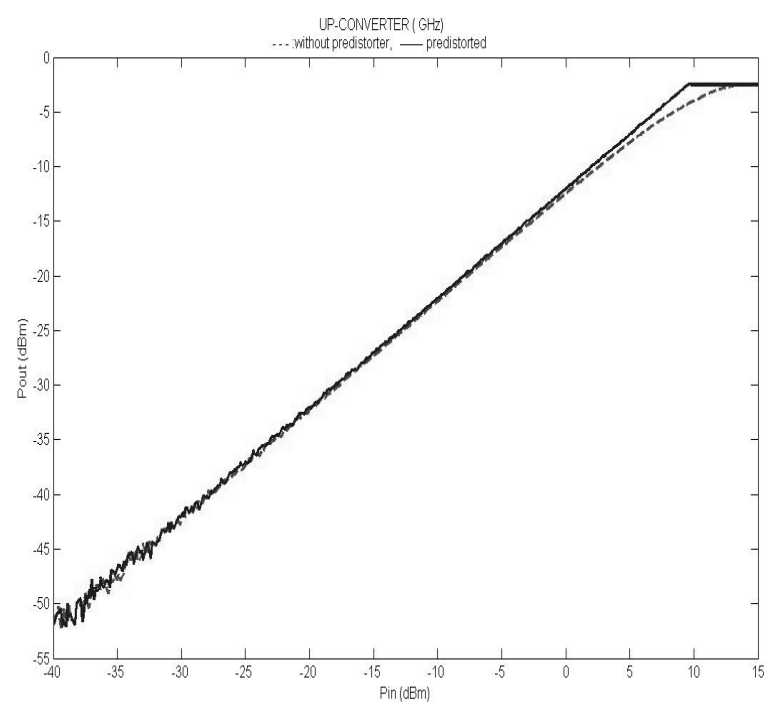

Fig.7. Linearized and unlinearized up-converter AM-AM characteristics.

\section{CONCLUSION}

Up to now some analog techniques, based on different kind of signal cancellation loop were used for mixer linearization. Thus paper contributes with the adoption of a basic digital predistorter. In spite that only AM-AM distortion is predistorted, results show an enlargement of $10 \mathrm{~dB}$ in the linear gain of the up-converter. The low-complexity of the predistortion strategy is allows its allocation is already existing DSP/FPGA devices without interference with other functionalities.

\section{ACKNOWLEDGEMENT}

This work was supported by the EU network TARGET "Top Amplifier Research Group in a European Team" (IST-1507893-NOE), by the EU project CAPANINA "Communications from Aerial Platform Networks delivering Broadband for ALL"(IST-2003-506745) and by the Spanish Government (CICYT project TEC2005-07985-C03-02).

\section{REFERENCES}

[1] T. J. Ellis, "A modified feed-forward technique for mixer linearization," in IEEE MTT-S Int. Microwave Symp. Dig., 1998.

[2] Potter, A.; Warr, P.A.; McGeehan, J.P."Efficient mixer linearisation scheme exploiting inherent error signals", Elect. Letters, vol. 40, n. 20, Sept. 2004

[3] Nesimoglu, T.; Beach, M.A.; MacLeod, J.R.; Warr, P.A,'Mixer linearisation for software defined radio applications", Vehicular Technology Conference, 2002. VTC 2002, Vol. 1, Sept. 2002.

[4] Y. W. Kim, Y. S. Kim, and S. H. Lee, "Linearized mixer using predistortion technique," IEEE Microwave and Wireless Comp. Lett., vol. 12, no. 6, June 2002

[5] Cheng, K.-K.M.; Chung-Fai Au-Yeun, "Novel difference-frequency dualsignal injection method for CMOS mixer linearization", Microwave and Wireless Comp. Letters, Vol. 14, n.. 7, July 2004 ,

[6] A.J. Zozaya, E. Bertran, "On the Performance of Cartesian Feedback and Feedforward Linearization Structures operating al Low Wavelengths". IEEE Trans. on Broadcact. Vol .50, n. 4 , Dec. 2004.

[7] S. Boumaiza, F.M. Ghannouchi, "Realistic power amplifierscharacterization with application to baseband digital predistortion for 3G base stations," IEEE Trans. on MTT, , vol. 50, n.. 12 Dec. 2002.
[8] P.L. Gilabert, G. Montoro and E. Bertran , "On the Wiener and Hammerstein Models for Power Amplifier Predistortion" AsiaPacific Microwave Conference, APMC 2005, Suzhou, China.

[9] P. Salmi, M. Neri and G. Corazza, "Design and Performance of Predistortion Techniques in Ka-band Satellite Networks", 22nd AIAA International Communications Satellite Systems Conf. (ICSSC), Monterey (Ca), May 2004. 